\title{
TWO TYPES OF PHASE SYNCHRONIZATION DESTRUCTION
}

\author{
Alexander E. Hramov, Alexey A. Koronovskii, and Maria K. Kurovskaya \\ Faculty of Nonlinear Processes, Saratov State University, Astrakhanskaya, 83, Saratov, 410012, Russia
}

(Dated: October 1, 2018)

\begin{abstract}
In this article we report that there are two different types of destruction of the phase synchronization regime of chaotic oscillators depending on the parameter mismatch as well as in the case of the classical synchronization of periodic oscillators. When the parameter mismatch of the interacting chaotic oscillators is small enough the PS breaking takes place without the destruction of the phase coherence of chaotic attractors of oscillators. Alternatively, due to the large frequency detuning, the PS breaking is accomplished by loss of the phase coherence of the chaotic attractors.
\end{abstract}

PACS numbers: 05.45.-a, 05.45.Xt, 05.45.Tp

Keywords: coupled oscillators, chaotic synchronization, phase synchronization, Lyapunov exponents

\section{Introduction}

Phase synchronization (PS) of chaotic oscillators observed in many systems (physical, technical, chemical, biological, physiological, etc.) has attracted great attention of scientists recently [1, 2, 3]. PS has practical importance [4, 5], with the phase synchronization concept involving the problems of the phase coherence of chaotic attractor 6, 7] and occurrence (destruction) of PS regime [8, 9].

The scientists usually do not distinguish between types of the phase synchronization regime destruction. As an exception we can mention work [9] where the existence of three types of transitions to PS regime in the coupled oscillators depending on the coherence properties of oscillations were described. Contrary to the conclusion obtained in [9], in this paper we report that there are two different types of the PS regime destruction depending on the detuning of the system control parameters as well as in the case of the classical synchronization of the periodic oscillators. We find the following: that when the parameter mismatch of the interacting chaotic oscillators is small enough the PS breaking takes place without destruction of the phase coherence of chaotic attractors of oscillators, whereas for the large parameter detuning with the decrease of the coupling strength between oscillators (or the amplitude of the external signal) the PS breaking is accompanied by the loss of the phase coherence of the chaotic attractors. To make our arguments more convenient we compare our methods and results with the ones described in work [9] .

The structure of this paper is the following. Section [I presents two scenarios of the destruction of the phase synchronization regime in the unidirectionally coupled Rössler oscillators. Section II explains the mechanisms resulting in these scenarios of the phase synchronization destruction. Section III shows the relationship between Lyapunov exponents and the phase synchronization boundary. Section IV displays the phase synchronization regime destruction in the systems with initially incoherent chaotic attractors. Section $\mathrm{V}$ provides the final conclusion.

\section{DESTRUCTION OF PHASE SYNCHRONIZATION REGIME IN TWO UNIDIRECTIONALLY COUPLED RÖSSLER SYSTEMS}

Let us start our consideration with two unidirectionally coupled Rössler systems

$$
\begin{aligned}
& \dot{x}_{d}=-\omega_{d} y_{d}-z_{d}, \\
& \dot{y}_{d}=\omega_{d} x_{d}+a y_{d}, \\
& \dot{z}_{d}=p+z_{d}\left(x_{d}-c\right), \\
& \dot{x}_{r}=-\omega_{r} y_{r}-z_{r}+\varepsilon\left(x_{d}-x_{r}\right), \\
& \dot{y}_{r}=\omega_{r} x_{r}+a y_{r}, \\
& \dot{z}_{r}=p+z_{r}\left(x_{r}-c\right),
\end{aligned}
$$

where $\varepsilon$ is a coupling parameter. The control parameter values have been selected by analogy with [10, 11] as $a=0.15, p=0.2, c=10.0$. The $\omega_{r}$-parameter which determines the main frequency of the response system has been selected as $\omega_{r}=0.95$, and the analogous parameter $\omega_{d}$ of the drive system has been varied in the range from 0.8 to 1.1 providing the mismatch of the interacting oscillators. The mentioned above control parameter values provide the phase coherence of chaotic attractors of both drive and response systems for zero coupling strength.

In Fig. 1 the phase synchronization area on the parameter plane $\left(\omega_{d} ; \varepsilon\right)$ is shown. Following to Ref. [9] we use two criteria to detect the presence of the phase synchronization regime. First, in the regime of the phase synchronization, locking of the mean frequencies

$$
\Omega_{d}=\left\langle\frac{d \varphi_{d}(t)}{d t}\right\rangle=\Omega_{r}=\left\langle\frac{d \varphi_{r}(t)}{d t}\right\rangle
$$

should take place, where $\langle\cdot\rangle$ denotes the time average, $\varphi_{d}$ and $\varphi_{r}$ are instantaneous phases of drive and response oscillators, respectively. Second, the presence of PS may be detected by means of the examination of the phase locking condition [1, 2, 3, 12 ]

$$
\left|\varphi_{d}(t)-\varphi_{r}(t)\right|<\text { const },
$$

with the instantaneous phase of chaotic signal being introduced in a traditional way as the rotation angle $\varphi=\arctan (y / x)$. 


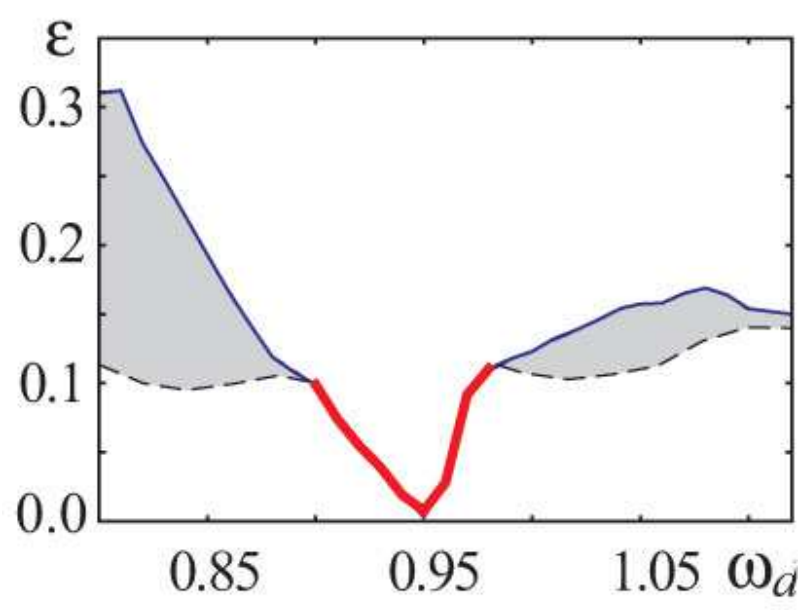

FIG. 1: (Color online) The PS area on the $\left(\omega_{d}, \varepsilon\right)$-plane. The PS boundary is shown in the range of the small parameter mismatch (the bold solid line) of coupled oscillators as well as in the range of the large one (the thin solid line). The chaotic attractor is incoherent in the region shown in grey color. The lower bound of the attractor non-coherence area is shown by the dashed line

Examining the behavior of two unidirectionally coupled Rössler systems (11) we have found two different types of the PS regime destruction. When the parameter mismatch of the interacting chaotic oscillators is large enough, the PS breaking is accompanied by the loss of the phase coherence of the chaotic attractors, whereas for the small parameter detuning with the decrease of the coupling strength between oscillators PS breaking takes place without destruction of the phase coherence of chaotic attractors of oscillators. According to [1, 3] the attractor is phase coherent when the phase trajectory rotates around the origin of the projection plane without crossing it. Alternatively, the attractor is supposed to be incoherent.

In Fig 2, $a, b$ the chaotic attractors of the response Rössler system and the dependence of the phase difference $\varphi_{d}(t)-\varphi_{r}(t)$ on time $t$ are shown below and above the onset of the PS regime for the small mismatch of $\omega$ parameters. One can easily see that with the decrease of the coupling strength $\varepsilon$ the PS regime is destroyed, with the chaotic attractor remaining phase coherent. For the large mistuning of the system control parameters the different scenario of the PS destruction takes place (see Fig. 2, $c, d)$. Below the boundary of the PS area the chaotic attractor of the response Rössler system loses its phase coherence.

Since the notion of the attractor coherence plays the key role in the chaotic synchronization theory the quantitative measure for the phase coherence should be defined. The measure of coherence may be characterized by means of the minimal distance $\rho$ between the points of the phase
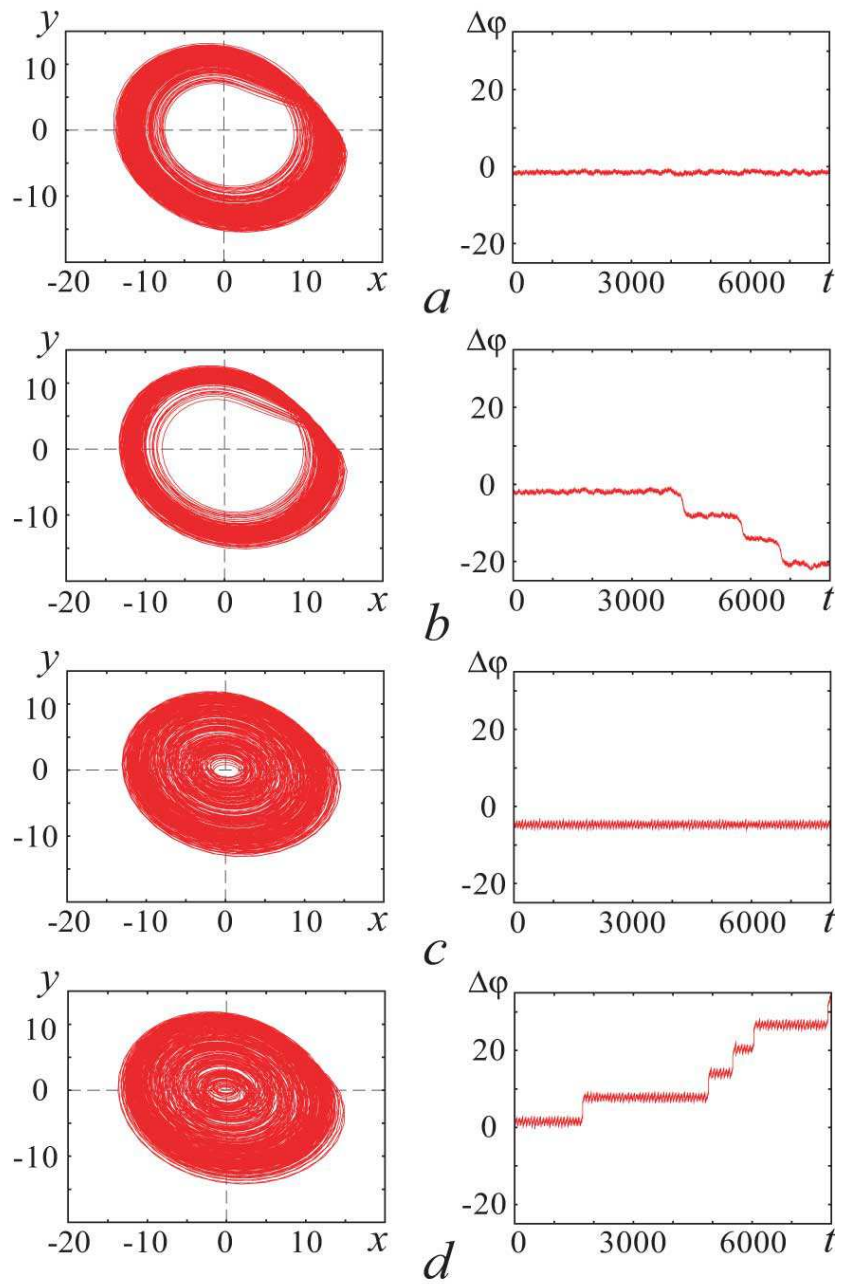

FIG. 2: (Color online) Attractors of the response Rössler system (11) and the dependencies of the phase differencies $\Delta \varphi(t)=\varphi_{d}(t)-\varphi_{r}(t)$ on time $t$ for small detuning $\left(\omega_{d}=\right.$ $0.91)$ of the drive Rössler system $(a)$ above $(\varepsilon=0.08)$ and $(b)$ below $(\varepsilon=0.075)$ the onset of the PS regime. Analogous figures for the large $\left(\omega_{d}=1.00\right)$ detuning of the $\omega_{d}$-parameter: (c) $\varepsilon$ is above $(\varepsilon=0.127)$ and $(d)$ below $(\varepsilon=0.123)$ the PS boundary

trajectory and the origin

$$
\rho=\min _{t \rightarrow+\infty} \sqrt{x^{2}(t)+y^{2}(t)},
$$

where $x(t), y(t)$ are the coordinates of the phase trajectory projection. Obviously, $\rho>0$ for the phase coherent chaotic attractor, and $\rho$ becomes equal to zero when attractor loses its coherence.

In Ref. [9] the coherence of chaotic attractor of the system under study was characterized by the sequence of the local maxima of $y(t)$, i.e. $\max (y)$. The chaotic attractor is incoherent when some $\max (y)<Y_{0}$ occurs, where $\left(X_{0}, Y_{0}\right) \approx(0,0)$ is the fixed point around which the phase trajectory rotates. Such criterion is limited, since only one coordinate is considered. Our definition (4) of 


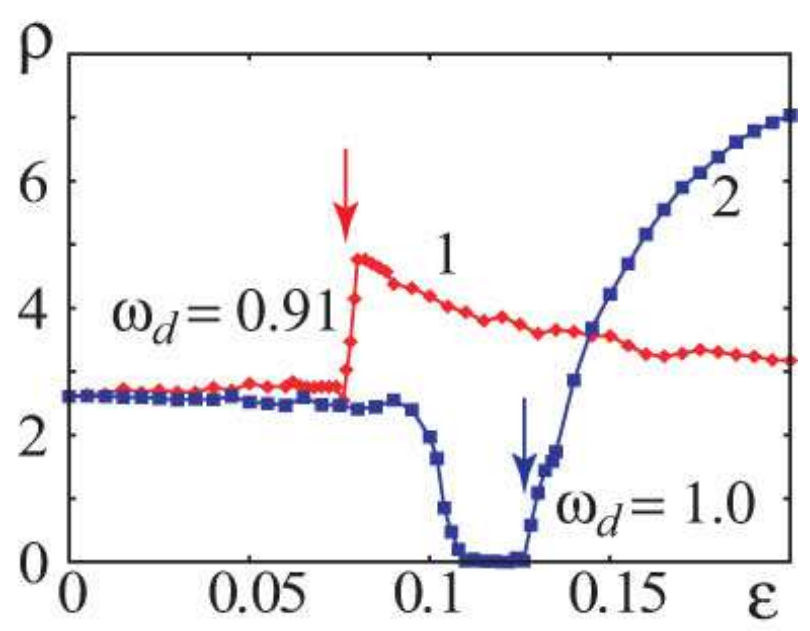

FIG. 3: (Color online) The dependencies of the coherence measure $\rho$ on the $\varepsilon$-parameter for the small (curve 1) and large (curve 2) system parameter mismatches. The arrows show the values of the coupling strength $\varepsilon$ corresponding to the PS destruction.

the coherence measure allows to generalize the approach used in [9] in such a way for both coordinates to be taken into account.

The dependencies of the coherence measure $\rho$ of the chaotic attractor of the response system on the coupling strength $\varepsilon$ are shown in Fig. 3 for both small (curve 1) and large (curve 2) parameter mismatches.

One can easily see that for small detuning of the control parameters (curve 1 in Fig. 31) the measure of the coherence $\rho$ is positive for all values of the coupling strength, whereas for the large parameter mistuning (curve 2 in Fig. (3) there is an area just below the PS boundary where the coherence measure is equal to zero. Therefore, it is evident, that the chaotic attractor loses its coherence when the PS regime is destroyed in the case of large parameter detuning and remains phase coherent both below and above the PS boundary if parameter mistuning is small enough.

The area on the plane $\left(\omega_{d}, \varepsilon\right)$ where the chaotic attractor of the response system is incoherent is shown in Fig. 1 in grey color. This region is joined closely to the boundary of the phase synchronization regime for the $\omega_{d}$-values detuned essentially from the $\omega_{r}$-parameter of the response Rössler system. Therefore, we can come to conclusion that depending on mistuning of the control parameters of system (11) the PS destruction occurs in two different ways, the mechanisms resulting to these types of PS destruction being supposed to be dissimilar.

Therefore, the different manifestations of these mechanisms may be observed in the vicinity of the PS boundary. In particular, two different types of the pre-transitional behavior revealed below the PS boundary may be considered as an indication of different mechanisms governing the scenarios of the phase synchronization destruc- tion. Indeed, it was found that the type-I intermittency and the super-long laminar behavior (so-called "eyelet intermittency" [13]) take place for small differences in the natural frequencies of the drive and response systems [13, 14, 15, 16, 17], while, as far as large values of the natural frequency differences are concerned, the ring intermittency emerges [18].

Let us show that the same both types of the synchronization destruction are also observed in the case of the driven periodic oscillators. This topic is discussed in detail in the next section.

\section{PHASE SYNCHRONIZATION DESTRUCTION OF VAN DER POL OSCILLATOR}

One of the approaches allowing to reveal different aspects of the synchronization phenomenon is the consideration of the periodic oscillators (see., e.g. [19, 20, 21, 22]). Therefore, let us use the classical model of the synchronization theory, namely, van der Pol oscillator

$$
\ddot{x}-\left(\lambda-x^{2}\right) \dot{x}+x=A \sin \left(\omega_{e} t\right)
$$

driven by the external harmonic signal with the amplitude $A$ and frequency $\omega_{e}$ to explain the mechanisms causing the PS regime destruction in the coupled Rössler systems (11). The value of the control parameter has been selected as $\lambda=0.1$. Oscillations in this case are certainly not chaotic but we can use the concepts of phase synchronization and phase coherence of an attractor in the same way as it is done for the chaotic oscillators. The phase of van der Pol oscillator is introduced as the rotation angle $\varphi=\arctan (y / x)$ as well as for the Rössler systems (11) considered above, whereas the phase of the external signal has been calculated as $\varphi_{e}(t)=\omega_{e} t$.

The synchronization area on the plane $\left(\omega_{e} ; A\right)$ is shown for the driven van der Pol oscillator (5) in Fig. 4. As well as for the unidirectionally coupled Rössler systems (1) described in Sec. [I two scenarios of the phase synchronization destruction may be observed. These two scenarios are illustrated in Fig [5. The attractors of van der Pol oscillator driven by the external harmonic signal (5), and the dependencies of the phase difference $\Delta \varphi(t)=\varphi(t)-\varphi_{e}(t)$ on time $t$ are shown in Fig. 5 , $a, b$ for the small frequencies detuning $\left(\omega_{0}-\omega_{e}\right)$ below and above the onset of the PS regime (here $\omega_{0}=\langle\dot{\varphi}(t)\rangle \simeq 1.0$ is the natural frequency of the autonomous van der Pol oscillator). Evidently, with the decrease of the amplitude of the external signal $A$ the PS regime is destroyed, with the attractor remaining phase coherent. Alternatively, for large mistuning of the frequencies, the different scenario of the PS destruction takes place (see Fig. $5, c, d$ ), since the attractor loses its phase coherence below the boundary of the PS area.

This statement may also be confirmed by means of the dependencies of the coherence measure $\rho$ of the attractor of the driven van der Pol oscillator on the amplitude $A$ 


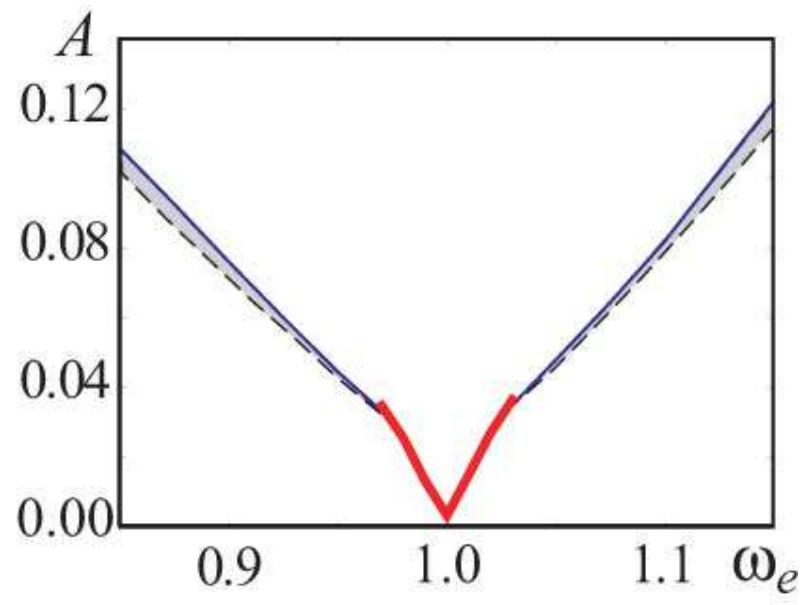

FIG. 4: (Color online) The PS area on the $\left(\omega_{e}, A\right)$-plane for the driven van der Pol oscillator. The PS boundary is shown in the range of the small frequency mismatch (the bold solid line) as well as in the range of the large one (the thin solid line). The attractor is incoherent in the narrow region shown in grey color. The lower bound of the attractor non-coherence area is shown by the dashed line

of the external signal given in Fig. 6 for both small and large frequencies mismatches $\left(\omega_{0}-\omega_{e}\right)$. Again, as in the case of coupled Rössler systems (Fig. 3) the measure of the coherence $\rho$ is positive for all values of the amplitude of the external signal (curve 1 in Fig. 6), if the frequencies $\omega_{0}$ and $\omega_{e}$ are detuned slightly, i.e., the attractor of the driven van der Pol oscillator remains phase coherent both below and above the PS boundary. As far as the large frequency mistuning is considered (curve 2 in Fig. 6) there is a narrow range located just below the PS boundary on which the coherence measure $\rho$ is equal to zero, hence the attractor loses its phase coherence, when the PS regime is destroyed.

The area on the plane $\left(\omega_{e}, A\right)$, where the attractor of the driven van der Pol oscillator (5) is incoherent, is shown in Fig. 4 in grey color. As in the case of Rössler systems (11) considered in Sec. I this region is joined closely to the boundary of the phase synchronization regime (although the width of this area is less than in the case of Rössler oscillators) when the frequency of the external signal is detuned essentially from the natural frequency of van der Pol oscillator.

Thus, when the PS regime is destroyed, the evolution of the attractor of van der Pol oscillator (5) driven by the external harmonic signal is the same as the evolution of the attractor of the response Rössler (11) system with a decrease of the coupling strength $\varepsilon$, despite of the fact that the Rössler system dynamics is chaotic, and van der Pol oscillator one - periodic. Therefore, we can assume that the destruction of the phase synchronization regime is governed by the same mechanisms in both cases, with transition from synchronous to asynchronous state of van der Pol oscillator being possible to be explained
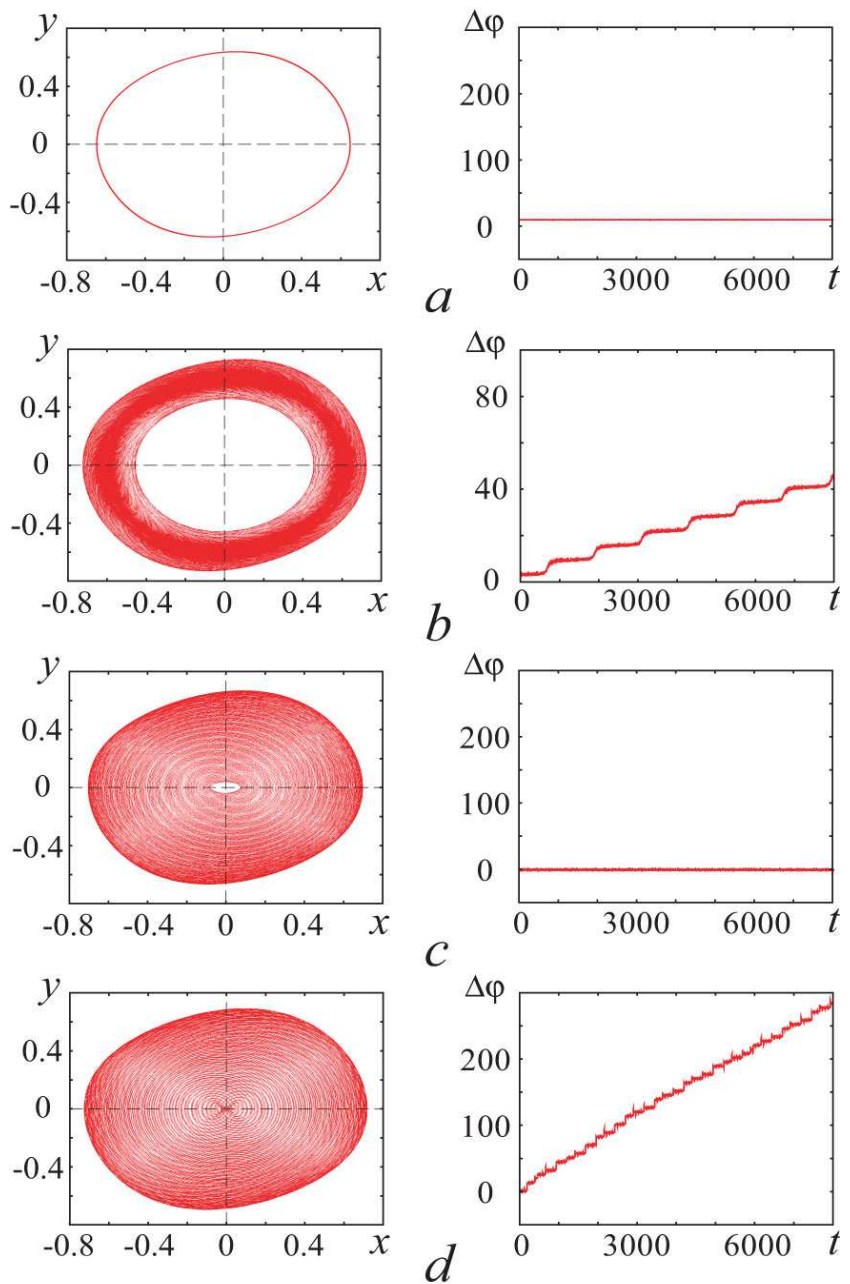

FIG. 5: (Color online) Attractors of van der Pol oscillator driven by the external harmonic signal (5) and the dependencies of the phase difference $\Delta \varphi(t)=\varphi(t)-\omega_{e} t$ on time $t$ for small $\left(\omega_{e}=0.98\right)$ frequency detuning $(a)$ above $(A=0.0250)$ and $(b)$ below $(A=0.0230)$ the onset of the PS regime. Analogous figures for the large $\left(\omega_{e}=0.9\right)$ detuning of the external signal frequency: $(c) A$ is above $(A=0.0775)$ and $(d)$ below $(\mathrm{A}=0.0750)$ the PS boundary

analytically by means of the complex amplitude method.

It is well known that there are two different scenarios for synchronization destruction in a periodic oscillator driven by an external force, corresponding to small and large detunings between the natural and external signal frequencies (see, e.g., tutorial [12]), respectively. Under certain conditions (i.e., for the periodically forced weakly nonlinear isochronous oscillator), the complex amplitude method may be used to find the solution describing the oscillator behavior in the form

$$
x(t)=\operatorname{Re} a(t) e^{i \omega t} .
$$

For the complex amplitude $a(t)$ one obtains the averaged (truncated) equations

$$
\dot{a}=-i \nu a+a-|a|^{2} a-i k,
$$




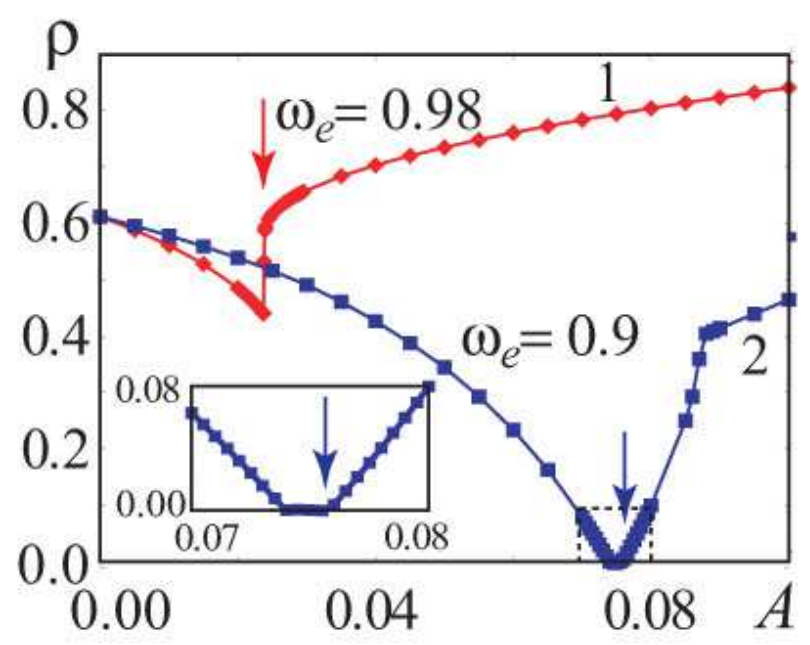

FIG. 6: (Color online) The dependence of the coherence measure $\rho$ on the $A$-parameter for the small (curve 1, $\omega_{e}=0.98$ ) and large (curve $2, \omega_{e}=0.9$ ) frequency mismatches. The arrows show the values of $A$-parameter corresponding to the PS destruction. In the frame the small part of the $\rho(A)$ dependence near the point of the PS destruction for the large frequency detuning is shown

where $\nu$ is the frequency mismatch, and $k$ is the (renormalized) amplitude of the external force. For the small $\nu$ and large $k$ the stable fixed point on the plane of the complex amplitude

$$
a^{*}=A e^{j \phi}=\mathrm{const}
$$

corresponds to the synchronous regime, with the synchronization destruction corresponding to the local saddlenode bifurcation associated with the global bifurcation of the limit cycle birth. Note, in this case the amplitude of the originated limit cycle is large initially. For the large frequency mismatches, the different mechanism of synchronization destruction is observed. With the decrease of $k$-value the fixed point (stable node) on the complex amplitude plane becomes sequentially a stable focus and an unstable focus (via the Andronov-Hopf bifurcation), with the limit cycle originating with the infinitesimal amplitude. With the further decrease of $k$-parameter, the amplitude of the limit cycle grows from zero monotonically. In this case, the phase synchronization destruction is connected with the limit cycle location on the complex amplitude plane [12]. When the limit cycle starts enveloping the origin, the synchronization regime begins to be destroyed. Obviously, in this case the attractor loses its phase coherence due to the fact that there are the moments of time when the modulus of amplitude $|a(t)|$ is equal to zero. As far as the small frequency mismatch is concerned, the attractor is always phase coherent due to $|a(t)| \neq 0, \forall t \in(-\infty ;+\infty)$.

Since the use of Eq. (6) for application of the complex amplitude method is equivalent to the transition to the revolving coordinate system, two mechanisms of the
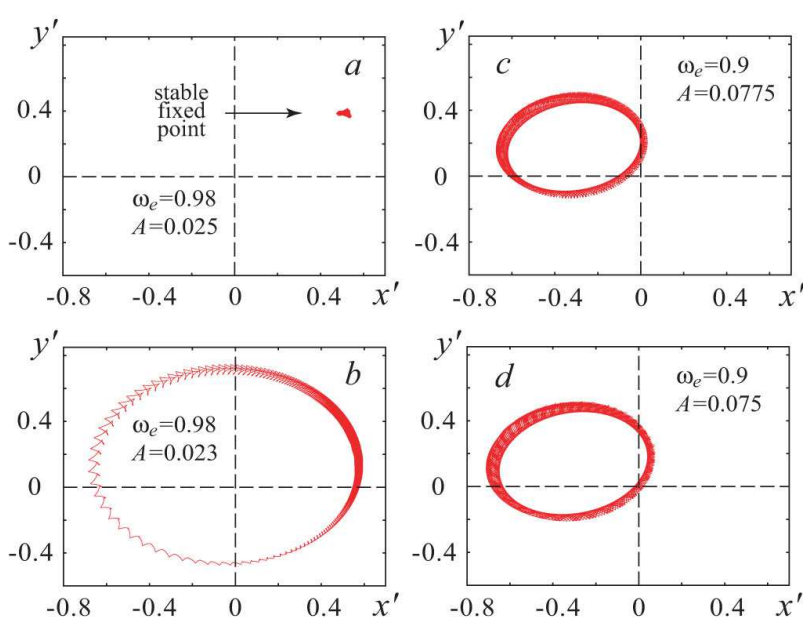

FIG. 7: (Color online) The phase trajectories of driven van der Pol oscillator on the rotating plane $\left(x^{\prime}, y^{\prime}\right)$ for the small $(a, b)$ and large $(c, d)$ parameter mistuning, the $A$-values having been selected just above $(a, c)$ and below $(b, d)$ the PS boundary. Figures $(a, c)$ correspond to the synchronization regime, figures $(b, d)$ illustrate the asynchronous behavior

phase synchronization destruction described above may be easily revealed by means of the consideration of the behavior of the driven oscillator on the plane $\left(x^{\prime}, y^{\prime}\right)$ rotating with the frequency $\omega_{e}$ of the external signal around the origin. These considerations of the rotating plane may be made apparent by using the coordinate transformation

$$
\begin{aligned}
& x^{\prime}=x_{r} \cos \varphi+y_{r} \sin \varphi, \\
& y^{\prime}=-x_{r} \sin \varphi+y_{r} \cos \varphi,
\end{aligned}
$$

where $\varphi=\varphi_{e}(t)$ is the instantaneous phase of the external signal.

In case of small frequency detuning $\left(\omega_{0}-\omega_{e}\right)$ the stable node is observed on the rotating plane $\left(x^{\prime} ; y^{\prime}\right)$ for the synchronized van der Pol oscillator (Fig. 7 $a$ ). As soon as the amplitude of the external signal starts to be below the phase synchronization boundary, the local saddle-node bifurcation, associated with the global bifurcation of the limit cycle birth, takes place and on the $\left(x^{\prime}, y^{\prime}\right)$-plane the cycle with the initially large amplitude is becoming observable (Fig. 7, $b$ ) instead of the stable node. As far as the large frequency difference $\left(\omega_{0}-\omega_{e}\right)$ is concerned a cycle (may be smeared, if the $\lambda$-parameter is not small enough) should be found on the rotating plane both below and above the PS synchronization boundary (Fig. 7, $c, d$ ). With the decrease of the intensity of the external signal the amplitude of the cycle increases monotonically, with the destruction of the phase synchronization regime corresponding to the control parameter value when the cycle starts to cross the origin (Fig. 7, $d$ ). When the cycle does not envelop the origin (see Fig.7 $c$ ), the phase synchronization regime takes place.

Obviously, if the mechanisms causing the PS regime destruction are the same in the cases of both periodic and 

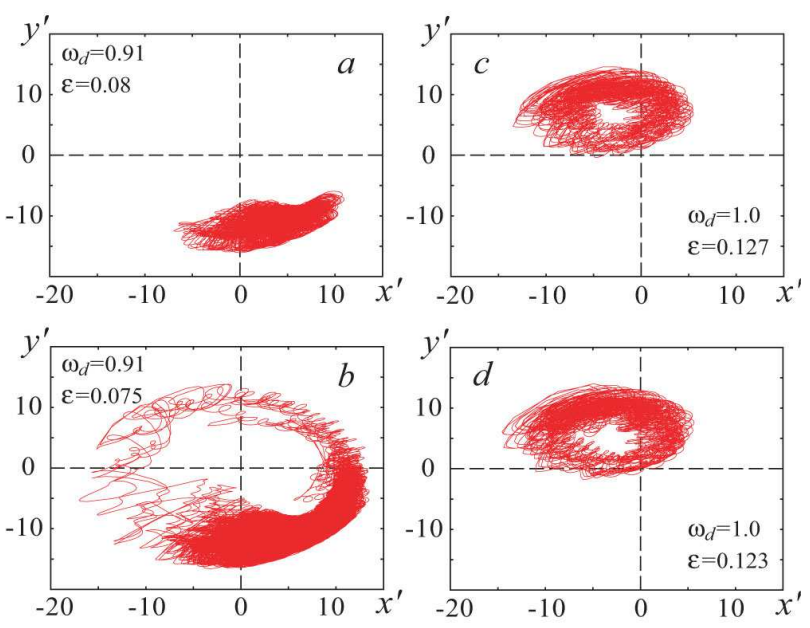

FIG. 8: (Color online) The phase trajectories of the response Rössler system (1) on the rotating plane $\left(x^{\prime}, y^{\prime}\right)$ for small $(a, b$, $\left.\omega_{d}=0.91\right)$ and large $\left(c, d, \omega_{d}=1.0\right)$ parameter mistuning, the coupling strength values having been selected just above $(a, c)$ and below $(b, d)$ the PS boundary

chaotic oscillators, one has to obtain the similar results for chaotic oscillators, too. Therefore, let us consider the dynamics of coupled Rössler systems (1) on the rotating plane (9) in the same way as it was done for the driven van der Pol oscillator, with the phase $\varphi=\varphi_{d}(t)$ of the drive Rössler system being used instead of the phase of the harmonic signal.

The results obtained for Rössler systems (1) are presented in Fig. 8, For the small system parameter mismatch (Fig. 8, $a$ ) the behavior of the synchronized response oscillator in the vicinity of the PS boundary looks like noise smeared stable fixed point on the $\left(x^{\prime}, y^{\prime}\right)$-plane. This effect arises insofar as the Rössler system may be considered as a noise smeared periodic oscillator (see, e.g., [23]). When the coupling strength between Rössler oscillators decreases and the PS regime is destroyed, the stable noise smeared fixed point disappears and, in full agreement with the dynamics of the driven periodic oscillator (compare with Fig. 7 $a, b$ ), the noise smeared limit cycle with initially large amplitude occurs (Fig. 8, b). Similarly, as well as in the case of van der Pol oscillator, if the large parameter mismatches are concerned, the noise smeared cycle is observed both below and above the PS regime boundary (see Fig. 8, $c, d$ and compare it with Fig. 7, $c, d)$, with the destruction of the phase synchronization regime corresponding to the cycle starting enveloping the origin (Fig. 8, $d$ ).

Thus, taken into account the results mentioned above we can draw a conclusion that the mechanisms of two types of the chaotic PS destruction are the same as in the case of the synchronization of periodic oscillations.

\section{LYAPUNOV EXPONENTS AND PHASE SYNCHRONIZATION BOUNDARY}

Let us discuss now the problem concerning the relationship between the observed types of PS destruction and transitions to PS regime described in Ref. 9]. In 9] three different types of transitions from asynchronous dynamics to the phase synchronization regime were reported of, depending on the coherence properties of the chaotic attractors of the uncoupled systems.

The degree of noncoherence of attractors of the autonomous oscillators was evaluated in [9] by means of the phase diffusion coefficient

$$
\left\langle(\varphi(t)-\langle\varphi(t)\rangle)^{2}\right\rangle=2 D_{\varphi} t
$$

where $\varphi(t)$ is the instantaneous phase of chaotic oscillator, $\langle\cdot\rangle$ denoting the ensemble average. For a phase coherent chaotic attractor the phase increases approximately uniformly and the value of $D_{\varphi}$ is rather small, whereas for a funnel attractor the increase of the phase is strongly nonuniform and $D_{\varphi}$ is a few orders larger in magnitude.

As a criterion of distinction of different types of transitions to/from the PS regime, the correlation between the dependency of the PS synchronization threshold (the critical curve $l_{1}$ ) and the dependencies of Lyapunov exponents (LEs) (the critical curve $l_{2}$ corresponds to the transition of one of the zero Lyapunov exponents to the negative values and the critical curve $l_{3}$ corresponds to passing through zero of the one of the positive Lyapunov exponents) on the coupling strength has been used. The types of transitions described in [9] were the following.

(I) Both uncoupled oscillators are characterized by the phase coherent chaotic attractors, therefore the value of the diffusion of phase $D_{\varphi}$ is small (e.g., $D_{\varphi} \sim 10^{-3}$ for the system considered in [9]). In this case, the phase synchronization regime is assumed to occur immediately after the transition of one of the zero Lyapunov exponents to a negative value, i.e., the critical curves $l_{1}$ and $l_{2}$ are close to each other, curve $l_{2}$ preceding curve $l_{1}$.

(II) Both chaotic attractors of the uncoupled oscillators are funnel, and the phase diffusion takes the intermediate values in comparison with types I and III. In [9] the values of $D_{\varphi} \sim 10^{-3} \div 10^{-1}$ were reported for this type of transitions. The critical curves $l_{1}$ and $l_{2}$ are supposed to be clearly separated, both lying below the critical curve $l_{3}$.

(III) The uncoupled oscillators considered are characterized by highly incoherent chaotic attractors, and the phase diffusion is rather strong (for the system considered in [9] $D_{\varphi}$ exceeds $10^{-1}$ ). In this case, the critical curve $l_{1}$ is expected to lie above both curves $l_{2}$ and $l_{3}$, i.e., the phase synchronization regime occurs after one of the positive Lyapunov exponents passes to negative values.

So, Lyapunov exponents seem to be important to distinguish between different types of transitions to/from the phase synchronization regime. Moreover, PS regime 


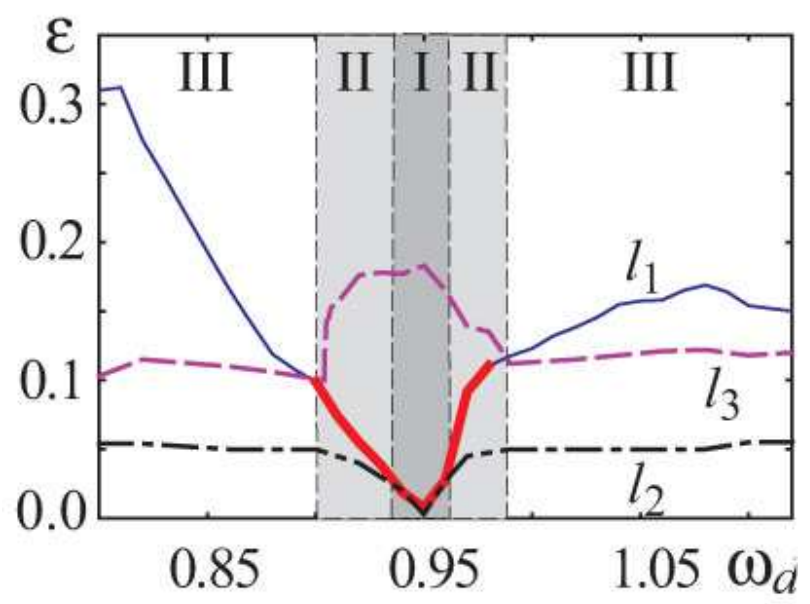

FIG. 9: (Color online) The critical curves $l_{1}, l_{2}$ and $l_{3}$ on the $\left(\omega_{d}, \varepsilon\right)$-plane. The critical curve $l_{1}$ corresponds to the PS boundary, it is shown in the range of the small parameter mismatch (the bold solid line) of coupled oscillators as well as in the range of the large one (the thin solid line). The dotted line $l_{2}$ corresponds to the transition of one of the zero Lyapunov exponents to negative values. The dashed line $l_{3}$ taken from our previous work [10] corresponds to zero crossing of the one of the positive Lyapunov exponents. The parameter plane is divided into three regions according to three types of transitions to phase synchronization described in 9] (regions I, II and III, respectively)

arising is usually described in terms of Lyapunov exponents (LEs) (see, e.g. [1, 2, 3, 29]). Eventually, we have to compare our results concerning different types of transitions from/to the PS regime with the ones given in [9]. Therefore, let us consider the relation between critical curves $l_{1}, l_{2}$ and $l_{3}$ corresponding to the onset of phase synchronization, the transition of one of the zero Lyapunov exponents to negative values and the passing of the one of the positive Lyapunov exponents through zero for the coupled Rössler oscillators (11) on the $\left(\omega_{d}, \varepsilon\right)$ parameter plane, respectively (see Fig. 9).

As it was mentioned above, the autonomous systems (11) under study with the pointed values of control parameters (and zero coupling strength) are characterized by the phase coherent attractors in the whole range of $\omega$-parameter variation. Correspondingly, the phase of chaotic signal increasing approximately uniformly, the phase diffusion coefficient $D_{\varphi}$ is rather small and does not exceed the value of $10^{-3}$ for all values of $\omega$-parameter. Therefore, we must expect that only the first type of transitions described in [9] is observed for two unidirectionally coupled oscillators (11), whereas two other types (II and III, respectively) can not be observed for this system. Nevertheless, having considered the dynamics of two unidirectionally coupled Rössler systems (1), we have observed types II and III of transitions as well as type I (see Fig. 9).

As it was mentioned above, the transition of one of the zero Lyapunov exponents to negative values is supposed to be closely connected with the onset of PS in the case when coupled oscillators are characterized by the phase coherent attractors (see also [8, 9] $)$. Our results refute this statement: Fig. 9 shows that the curve $l_{2}$ coincides with the curve $l_{1}$ (onset of PS) only in a very small range of the parameter mismatch of coupled oscillators (this range is shown in Fig. 9 in dark grey color and marked by number I), although uncoupled both the drive and response Rössler systems are characterized by the phase coherent attractors in the whole range of the $\omega_{d}$-parameter variation. We can not say that the transition of one of the zero LEs to negative values determines PS arising even in the case of small mismatch of system parameters. Indeed, for the parameter $\omega_{d}=0.9$ separating two types of the PS destruction the coupling strength value $\varepsilon_{P S} \approx 0.099$ corresponding to the onset of PS two times exceeds the $\varepsilon_{l_{2}}$-value corresponding to $l_{2}$-curve $\left(\varepsilon_{l_{2}} \approx 0.05\right)$ (see also Fig. (9). Therefore, we come to conclusion that the transition of one of the zero LEs to negative values precedes arising of the PS regime but does not determine it. We suppose that this transition may be connected with time scale synchronization [24, 25], but this problem requires further consideration.

Thus, despite the fact, that both interacting chaotic oscillators are characterized by the phase coherent attractors for zero coupling strength the first type of transitions described in Ref. 9] is observed only in the narrow range of the $\omega_{d}$-parameter values (see Fig. 9). Moreover, two other types of transitions, described in [9] and supposed to correspond to the synchronization of oscillators with initially incoherent chaotic attractors, are also observed in the considered system (11) of oscillators with originally coherent chaotic attractors. Indeed, in [9] it was reported that the case when the boundary of the PS regime arising (curve $l_{1}$ ) is between the critical curves $l_{2}$ and $l_{3}$ corresponds to the synchronization of oscillators with funnel chaotic attractors. Nevertheless, for the considered system (11) this type of transitions takes place in the $\omega_{d}$-parameter value ranges shown in Fig. 9 in light grey color and labeled by number II. Similarly, the third type of transitions described in [9] and supposed to correspond to the systems with highly incoherent attractors (the critical curve $l_{1}$ lies above both the critical curves $l_{2}$ and $l_{3}$ ) is also observed in (1) despite of the initial coherence of system attractors (white regions III in Fig. 9).

It should also be noted that the location of the critical curve $l_{3}$ on the $\left(\omega_{d} ; \varepsilon\right)$-plane coincides with the onset of the generalized synchronization (GS) regime [26, 27, 28]. The mechanisms determining the location of the GS boundary on the parameter plane have been considered for unidirectionally coupled Rössler systems (11) in our previous paper [10] with the help of the modified system approach [11]. Based on the results given in [10] we can make a decision that the location of the GS boundary (and, correspondingly, the critical curve $l_{3}$ ) does not relate with phase synchronization phenomenon.

Thus, we come to conclusion that for two unidirection- 
ally coupled Rössler oscillators (1) two types of the PS destruction are possible in dependence on the system parameter mismatch. Of course, one can distinguish three types of the PS destruction according to the location of the PS regime boundary and critical curves $l_{2}$ and $l_{3}$ on the parameter plane, but there is no reason of doing that. Contrary to distinguishing PS destruction types between two classes (when there are well-known physical mechanisms determining such approach), the consideration of three types of transitions to PS based on the locus of the critical curves $l_{1,2,3}$ on the parameter plane does not seem to be reasonable, since the positions of $l_{2,3}$ curves are caused by different mechanisms which do not concern the PS phenomenon.

\section{PHASE SYNCHRONIZATION DESTRUCTION IN THE CHAOTIC SYSTEMS WITH INITIALLY INCOHERENT CHAOTIC ATTRACTORS}

Since the behavior of unidirectionally coupled Rössler systems having been examined we came to conclusion that for the oscillators with initially phase coherent attractors (11) two types (rather than three) of the PS destruction should be distinguished, it is quite reasonable to learn what types of PS destruction may be observed if two oscillators with initially incoherent chaotic attractors are considered. To clarify this point we have studied two mutually coupled Rössler systems

$$
\begin{aligned}
& \dot{x}_{1,2}=-\omega_{1,2} y_{1,2}-z_{1,2}, \\
& \dot{y}_{1,2}=\omega_{1,2} x_{1,2}+a y_{1,2}+d\left(y_{2,1}-y_{1,2}\right), \\
& \dot{z}_{1,2}=0.1+z_{1,2}\left(x_{1,2}-8.5\right)
\end{aligned}
$$

from the point of view of the concept discussed in Sec. [1] and II. Note, it is the system that were considered in [9], for which three types of transitions connected with the attractor coherence properties and critical curves location had been described (Fig. 10).

The control parameter values of the systems (11) have been selected the same as they were given in [9]. In Eq. (11) $d$ is the coupling strength, $\omega_{1}=0.98, \omega_{2}=1.02$, the parameter $a \in[0.15 ; 0.3]$ determines the topology of chaotic attractors. It is known, that when $a$ exceeds the critical value $a_{c}\left(a_{c 1} \approx 0.186\right.$ for $\omega_{1}$ and $a_{c 2} \approx 0.195$ for $\left.\omega_{2}\right)$ the chaotic attractor of autonomous Rössler system becomes incoherent [9]. Following [9] the phase $\varphi(t)$ has been defined as the rotation angle $\varphi=\arctan (\dot{y} / \dot{x})$ on the plane $(\dot{x} ; \dot{y})$ to study PS of the systems with initially funnel attractors.

Having studied the behavior of two mutually coupled Rössler systems (11) along the boundary of PS regime on the plane $(a, d)$ given in [9], we have also found two scenarios of the PS regime destruction described in Sec. [I as well as for the case of two unidirectionally coupled oscillators (11). For small values of $a$-parameter $\left(a<a_{*} \approx 0.205\right)$ the first type of the PS regime destruction takes place when chaotic attractors of both systems

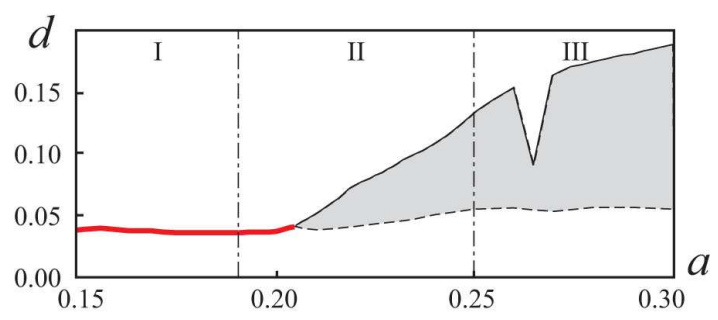

FIG. 10: The phase synchronization boundary of two coupled Rössler systems (11). The bold solid line corresponds to the phase synchronization regime destruction when both chaotic attractors retain their phase coherence both above and below the synchronization threshold. The thin line presents the synchronization boundary when the PS destruction is accompanied by break of the attractor coherence. The change of the type of the PS regime destruction takes place at $a_{*} \approx 0.205$. The attractor of the first Rössler system is incoherent in the region shown in grey color. The lower bound of the attractor non-coherence area is shown by the dashed line. The areas corresponding to three types of transitions to PS regime described in [9] are labeled by numbers I, II and III, respectively

keep their coherence on the plane $(\dot{x}, \dot{y})$ both above and below the boundary of PS (Fig. 10). When $a>a_{*}$ the chaotic attractor of the first system loses its coherence as soon as the coupling strength $d$ is below the onset of PS, with the attractor of the second system remaining to be phase coherent. Note, that $a_{*}>a_{c 1,2}$ therefore the change of the PS destruction type takes place when both coupled systems are characterized by the initially funnel chaotic attractors on the plane $(x, y)$.

To make our decision more convincing, we have also calculated the coherence measure $\rho$ vs. coupling parameter $d$ for two $a$-parameter values corresponding to both types of the PS regime destruction, to be exact $a_{I}=0.19<a_{*}$ and $a_{I I}=0.21>a_{*}$ (Fig. 11). Since the evolution of the chaotic attractors is considered on the velocity plane $(\dot{x}, \dot{y})$ we have used

$$
\rho=\min _{t \rightarrow+\infty} \sqrt{\dot{x}^{2}(t)+\dot{y}^{2}(t)}
$$

instead of Eq. (44). Evidently, the chaotic attractor of the first Rössler system remains phase coherent both below and above the PS boundary for $a_{I}=0.19$ and loses its coherence when the PS regime is destroyed in the case of $a_{I I}=0.21$.

The evolution of the chaotic attractor of the first Rössler system, when the PS regime is destroyed, is shown in Fig 12, The first type of the PS regime destruction, when the chaotic attractors are phase coherent both above and below the PS boundary, is shown in Fig $12 a, b$. Fig. 12, $c, d$ illustrates the second type of transitions accompanied by the destruction of the coherence of one of the chaotic attractors just below the PS boundary.

Thus, for two coupled systems with initially funnel attractors, the phase coherence of chaotic attractor on the velocity plane $(\dot{x}, \dot{y})$ is destroyed in the same way as it 


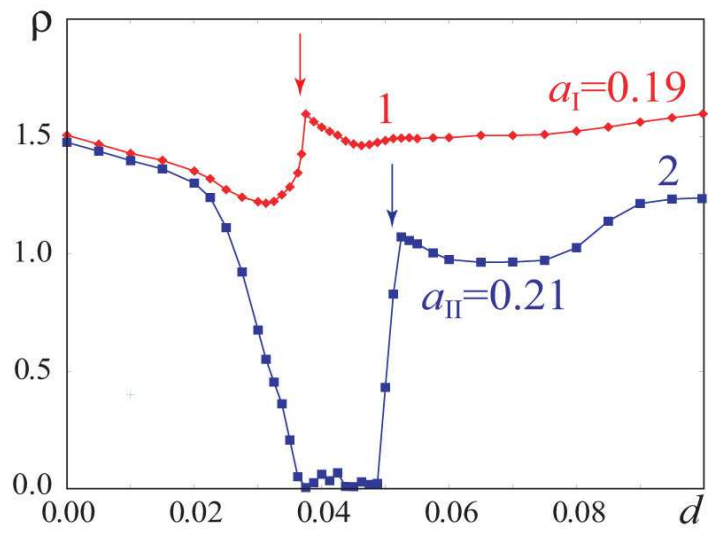

FIG. 11: (Color online) The dependencies of the coherence measure $\rho$ on the coupling strength $d$ for $a_{I}=0.19$ (curve 1) and $a_{I I}=0.21$ (curve 2). The arrows show the threshold of the PS regime

was observed on the plane $(x, y)$ for the systems (11) with initially phase coherent attractors.

Taking into account all obtained results we come to conclusion that for the system of two mutually coupled Rössler oscillators with initially phase incoherent attractors (11) as well as for the oscillators with phase coherent attractors (11) two types (instead of three) of the PS destruction should be distinguished. This conclusion is also confirmed by Fig. 6 given in [9] where the mean frequency ratio $\Omega_{1} / \Omega_{2}$ versus coupling strength is shown. The behavior of $\Omega_{1} / \Omega_{2}$ is quite different for the first and the second types of transitions to PS proposed in 9] but is the same for the second and the third ones.

\section{CONCLUSION}

We have described two different types of the PS regime destruction as well as the mechanisms resulting in the destruction, which are the same as in the case of the classical synchronization of periodic oscillators. The first of them is caused by the loss of the common rhythm of chaotic oscillations and the second one is caused by the loss of phase coherence of chaotic attractor. These types have been observed in the systems with initially both phase coherent and funnel chaotic attractors. The transition of one of the zero Lyapunov exponents to negative values precedes arising of the PS regime but does not cause it is the other important result. Similarly, the passing of the one of the positive Lyapunov exponent through zero does not relate to PS phenomenon. Therefore, there is no reason to use these LEs for the PS onset description.

We assume that the number of the possible transitions from the phase synchronization regime to the asynchronous dynamics might not be limited by two types only which are described in our paper. For the more complex systems (e.g., for the system with the larger dimen-
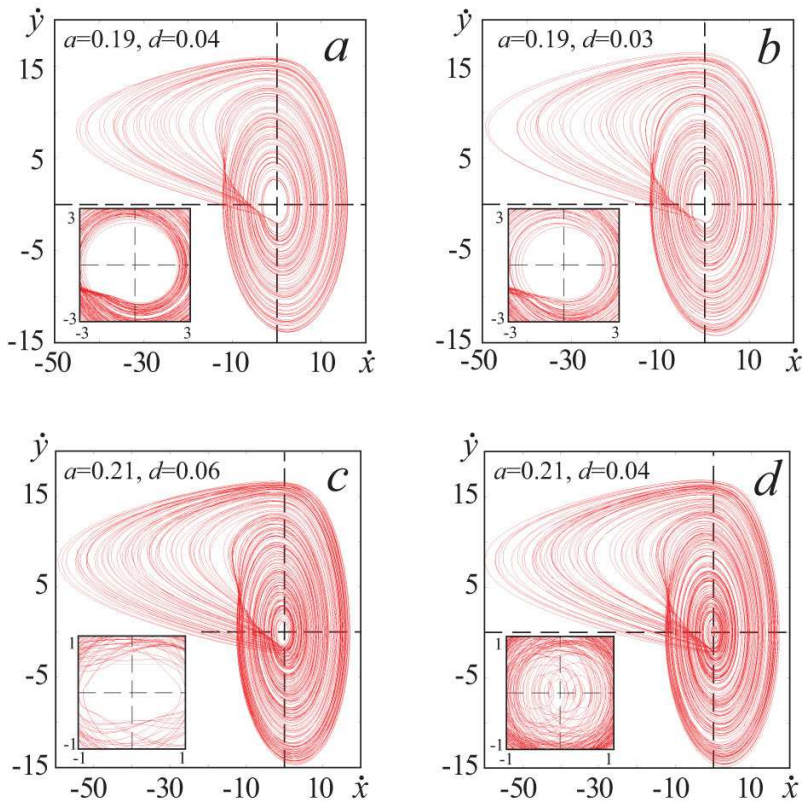

FIG. 12: (Color online) Attractors of the first Rössler system (11) for two values of $a$-parameter corresponding to two types of the PS regime destruction. Figures $(a, b)$ show the evolution of the system behavior on the velocity plane $(\dot{x}, \dot{y})$ for $a_{I}=0.19$ when the attractor remains phase coherent both above $(a)$ and below $(b)$ the PS regime boundary. The second scenario of the PS regime destruction $\left(a_{I I}=0.21\right)$ accompanied by the phase coherence loss of the chaotic attractor is shown in Fig. $(c, d)$. The behavior of coupled oscillators is synchronized in Fig. $(c)$ when the attractor of the first Rössler system on the plane $(\dot{x}, \dot{y})$ is phase coherent. Alternatively, the phase incoherent attractor is shown in Fig. $(d)$ after the PS regime has been destroyed. In the frames the trajectories on the plane $(\dot{x}, \dot{y})$ near the origin are shown.

sion of the phase space) the breakdown of PS may also be associated with global bifurcations, e.g., homoclinic orbits, Takens-Bogdanov bifurcation points, etc. [29]. Nevertheless, we suppose that the two considered types of the PS regime destruction should be typical for a wide class of nonlinear systems, e.g., such as Pierce diode 30. or laser system [16]. In particular, we have observed the same types of behavior in the vicinity of the PS boundary for the case of two unidirectionally coupled generators with tunnel diodes described in Ref. [31].

\section{Acknowledgment}

We thank Dr. S.V. Eremina for English language support. This work was supported by U.S. Civilian Research and Development Foundation for the Independent States of the Former Soviet Union (CRDF), grant REC-006, the Supporting program of leading Russian scientific schools (project NSh-4167.2006.2), RFBR (07-02-00044 and 0502-16273) and "Dynasty" Foundation. 
[1] A. S. Pikovsky, M. G. Rosenblum, and J. Kurths, Synchronization: a universal concept in nonlinear sciences (Cambridge University Press, 2001).

[2] V. S. Anishchenko, V. Astakhov, A. Neiman, T. E. Vadivasova, and L. Schimansky-Geier, Nonlinear Dynamics of Chaotic and Stochastic Systems. Tutorial and Modern Developments (Springer-Verlag, Heidelberg, 2001).

[3] S. Boccaletti, J. Kurths, G. V. Osipov, D. L. Valladares, and C. T. Zhou, Physics Reports 366, 1 (2002).

[4] R. QuianQuiroga, A. Kraskov, T. Kreuz, and P. Grassberger, Phys. Rev. E 65, 041903 (2002).

[5] M. G. Rosenblum, A. S. Pikovsky, J. Kurths, C. Schäfer, and P. A. Tass, in Handbook of Biological Physics (Elsiver Science, 2001), pp. 279-321.

[6] M. G. Rosenblum, A. S. Pikovsky, J. Kurths, G.V. Osipov, I.Z. Kiss, and J.L. Hudson, Phys. Rev. Lett. 89, 264102 (2002).

[7] V. S. Anishchenko and T. E. Vadivasova, Journal of Communications Technology and Electronics 49, 69 (2004).

[8] M. G. Rosenblum, A. S. Pikovsky, and J. Kurths, Phys. Rev. Lett. 78, 4193 (1997).

[9] G. V. Osipov, B. Hu, C. Zhou, M. V. Ivanchenko, and J. Kurths, Phys. Rev. Lett. 91, 024101 (2003).

[10] A. E. Hramov, A. A. Koronovskii, and O. I. Moskalenko, Europhysics Letters 72, 901 (2005).

[11] A. E. Hramov and A. A. Koronovskii, Phys. Rev. E 71, 067201 (2005).

[12] A. S. Pikovsky, M. G. Rosenblum, and J. Kurths, Int. J. Bifurcation and Chaos 10, 2291 (2000).

[13] A. Pikovsky, G. Osipov, M. Rosenblum, M. Zaks, and J. Kurths, Phys. Rev. Lett. 79, 47 (1997).

[14] A. S. Pikovsky, M. Zaks, M. G. Rosenblum, G. V. Osipov, and J. Kurths, Chaos 7, 680 (1997).

[15] K. J. Lee, Y. Kwak, and T. K. Lim, Phys. Rev. Lett. 81,
321 (1998).

[16] S. Boccaletti, E. Allaria, R. Meucci, and F. T. Arecchi, Phys. Rev. Lett. 89, 194101 (2002).

[17] E. Rosa, E. Ott, and M. H. Hess, Phys. Rev. Lett. 80, 1642 (1998).

[18] A. E. Hramov, A. A. Koronovskii, M. K. Kurovskaya, and S. Boccaletti, Phys. Rev. Lett. 97, 114101 (2006).

[19] A. G. Balanov, N. B. Janson, D. E. Postnov, and P. V. E. McClintock, Phys. Rev. E 65, 041105 (2002).

[20] P. Woafo and R. A. Kraenkel, Phys. Rev. E 65, 036225 (2002).

[21] A. G. Balanov, N. B. Janson, V. V. Astakhov, and P. V. E. McClintock, Phys. rev. E 72, 026214 (2005).

[22] A. E. Hramov, A. A. Koronovskii, V. I. Ponomarenko, and M. D. Prokhorov, Phys. Rev. E 73, 026208 (2006).

[23] A. S. Pikovsky, M. G. Rosenblum, G. V. Osipov, and J. Kurths, Physica D 104, 219 (1997).

[24] A. E. Hramov and A. A. Koronovskii, Chaos 14, 603 (2004).

[25] A. E. Hramov, A. A. Koronovskii, M. K. Kurovskaya, and O. I. Moskalenko, Phys. Rev. E 71, 056204 (2005).

[26] N. F. Rulkov, M. M. Sushchik, L. S. Tsimring, and H. D. I. Abarbanel, Phys. Rev. E 51, 980 (1995).

[27] H. D. I. Abarbanel, N. F. Rulkov, and M. M. Sushchik, Phys. Rev. E 53, 4528 (1996).

[28] K. Pyragas, Phys. Rev. E 54, R4508 (1996).

[29] D. E. Postnov, A. G. Balanov, N. B. Janson, and E. Mosekilde, Phys. Rev. Lett. 83, 1942 (1999).

[30] R.A. Filatov, A.E. Hramov, and A.A. Koronovskii, Phys. Lett. A 358, 301-308 (2006).

[31] M. G. Rosenblum, A. S. Pikovsky, and J. Kurths, IEEE Trans. Circuits and Syst. 44, 874 (1997). 